\title{
Dissolution Studies of Generic Medications: New Evidence of Deviations from the Transitivity Principle
}

\author{
M. Esperanza Ruiz ${ }^{1}$, Anabella Gregorini ${ }^{1}$, Alan Talevi ${ }^{2}$, and Maria G. Volonté ${ }^{1, *}$ \\ 'Quality Control of Medications, Department of Biological Sciences, Faculty of Exact Sciences, University of La Plata (UNLP), 47 \& 115, \\ B1900AJl, La Plata, Buenos Aires, Argentina \\ ${ }^{2}$ Medicinal Chemistry, Department of Biological Sciences, Faculty of Exact Sciences, University of La Plata (UNLP), Buenos Aires, Argentina
}

\begin{abstract}
Current international criteria to guarantee that two equivalent pharmaceutical medications are interchangeable establish that substitution may be done whenever studies indicate that a generic or similar product is bioequivalent to the reference product. The principle of transitivity defines interchangeability between approved pharmaceutical equivalents (i.e., given two generic products $B$ and $C$ and a reference $A$, if $B$ and $C$ are bioequivalent to $A$, then $B$ and $C$ are assumed to be bioequivalent although bioequivalence between them has not been actually assessed). In this study, the goal was to test the applicability of the transitivity principle in establishing interchangeability between pharmaceutical equivalents. We obtained the dissolution profiles of all the products corresponding to tablets of ranitidine $300 \mathrm{mg}$ and cephalexin 500 mg (Biopharmaceutics Classification System Class III), furosemide 40 mg (Class IV), and ibuprofen 400 mg and $600 \mathrm{mg}$ (BCS Class II) available in the Argentinean pharmaceutical market. Dissolution profiles of all possible pairs were compared through similarity factor $f_{2}$. The results obtained bring into question the general validity of the transitivity principle, as many examples were observed to have dissolution profiles similar to the reference product, yet were not equivalent. Although this has only been assessed through in vitro tests, our results are potentially important, as a number of recent reports have suggested the extension of biowaivers to certain BCS Class II and Class III products, including ranitidine hydrochloride and ibuprofen.
\end{abstract}

\section{INTRODUCTION}

\begin{abstract}
bout one third of the world population lacks access to essential medications and medical treatment (1). By 2000 , over $90 \%$ of the world pharmaceutical market was concentrated in only eight developed countries (Table 1) (1,2). Therefore, strategies to grant accessibility to medications to low-income patients are urgently needed. Among other policies aimed at regulating the price of medications (such as price control by the state), consolidation of a market of generic products has proved to be particularly effective; the price of a generic may range from $3 \%$ to $80 \%$ of the price of the innovator product, thus allowing drug consumers, health insurers, and governments to save a significant amount of money (3-6). A generic is an off-patent medication that has the same active ingredient, dose, and route of administration as the original product. Most countries require generic manufacturers to demonstrate that their formulation is within an acceptable bioequivalent range to a reference, brand-name counterpart (a product whose therapeutic and safety data are available), thus sharing, to some extent, pharmacokinetic and pharmacodynamic properties. Depending on the case, the reference product might be the innovator or the most frequently sold brand product $(7,8)$. Therefore, the establishment of bioequivalence is essential to interchangeability; that is, a patient can
\end{abstract}

${ }^{*}$ Corresponding author. substitute a generic for a particular product without jeopardizing efficacy or safety.

According to the FDA biowaiver guidance (9), a waiver for in vivo BE studies can be requested for solid, orally administered immediate-release products containing highly soluble and highly permeable drugs (Class I of the Biopharmaceutics Classification System, BCS) provided that the following requisites are met: the drug must be stable in the gastrointestinal tract, excipients do not affect the rate or extent of absorption, the drug must not have a narrow therapeutic index, and the drug product is designed not to be absorbed in the oral cavity. In those cases, the establishment of Class I status may replace a BE study. Recently, there has been intense discussion of the possibility of widening the eligibility for biowaivers to certain drugs of BCS Class II and III (10-12), including ibuprofen and other acidic NSAIDs $(13,14)$ and ranitidine (15). The WHO has presented relaxations for drug products containing BCS Class II and III active ingredients (16), and national regulatory agencies such as the Swedish MPA have approved biowaivers for drugs including ibuprofen, paracetamol, and prednisolone (12) . Although it has not yet been adopted by regulatory agents, the WHO has proposed extensions to the scope of biowaiver applications to BCS Class III drug products dissolving very rapidly at $\mathrm{pH} 1.2,4.5$, and 6.8 , BCS Class II weak acids with a dose-solubility ratio of $250 \mathrm{~mL}$ or less at $\mathrm{pH} 6.8$, and rapidly dissolving products with similar dissolution 
Table 1. Distribution of Global Expenditures on Medications by 2000 (Adapted from ref 2.)

\begin{tabular}{lcc}
\hline Country & $\begin{array}{c}\text { Expenditure (billions US } \\
\text { dollars) }\end{array}$ & $\begin{array}{c}\text { Percentage of global } \\
\text { expenditure }\end{array}$ \\
\hline USA & 149.5 & 52.9 \\
\hline Japan & 51.5 & 18.2 \\
\hline France & 16.7 & 5.9 \\
\hline Germany & 16.2 & 5.7 \\
\hline UK & 11.1 & 3.9 \\
\hline Italy & 10.9 & 3.9 \\
\hline Spain & 7.1 & 2.5 \\
\hline Canada & 6.2 & 2.2 \\
\hline Brazil & 5.2 & 1.8 \\
\hline Mexico & 4.9 & 1.7 \\
\hline
\end{tabular}

The ten countries with highest expenditures are presented.

profiles at $\mathrm{pH} 1.2,4.5$, and 6.8 (17). The risks of reaching an inappropriate biowaiver decision need to be evaluated more critically when the extent of absorption is low, the sites of absorption are restricted to the proximal regions of the gastrointestinal tract, and the mechanism of absorption is subject to induction or competition; in these cases, the excipients used should also be scrutinized carefully.

The principle of transitivity is assumed to be valid for defining interchangeability among all the approved pharmaceutical equivalents (i.e., given two generics $B$ and $C$ and a reference $A$, if $B$ is bioequivalent to $A$ and $C$ is bioequivalent to $A$, then $B$ and $C$ are assumed to be bioequivalent although bioequivalence between $B$ and C has not been actually proved), although it has been proved that the principle of transitivity does not always apply (18-20) (Figure 1).

In this study, we provide new evidence that the principle of transitivity is not generally applicable for defining interchangeability between pharmaceutical equivalents, based on exhaustive comparison of in vitro dissolution data from different medications from the Argentinean pharmaceutical market.

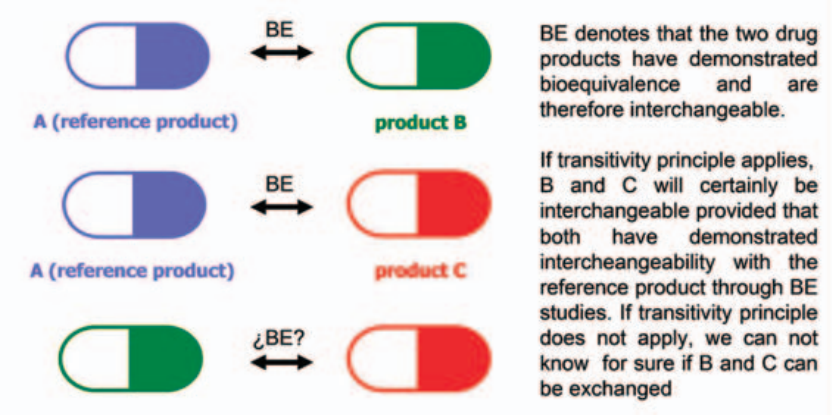

Figure 1. Schematic explanation of the transitivity principle.

\section{MATERIALS AND METHODS}

Equipment and Materials

All dissolution tests were conducted in a Sotax AT7 apparatus (Sotax AG, Basel, Switzerland), which is a manual-sampling dissolution bath with seven vessels to allow dissolution testing of six tablets at the same time and have fresh medium in another vessel to replenish after sampling. The amount dissolved was determined spectrophotometrically in a Thermo spectrophotometer, Helios Beta model (Thermo Fisher Scientific, Waltham, MA, USA). Drugs and reagents were weighed on a Mettler Toledo AG 204 balance (Metler, Greinfensee, Switzerland).

All medications assayed (ranitidine 300-mg, furosemide 40-mg, ibuprofen 400- and 600-mg, and cephalexin 500-mg tablets) were purchased at a local drugstore. Reference standards of all four drugs were purchased from the Argentinean National Institute of Medications (Buenos Aires, Argentina). All other chemicals used were of analytical grade, including potassium phosphate monobasic, hydrochloric acid, and sodium hydroxide.

\section{Dissolution Studies}

Dissolution profiles were obtained for all pharmaceutical products available in the Argentinean market for the following medications: ranitidine tablets $300 \mathrm{mg}$ (21 brands), furosemide tablets $40 \mathrm{mg}$ (11 brands), ibuprofen tablets $400 \mathrm{mg}$ (33 brands), ibuprofen tablets $600 \mathrm{mg}$ (12 brands), and cephalexin tablets $500 \mathrm{mg}$ ( 17 brands).

All dissolution tests were conducted according to USP 31 (21). Dissolution media were filtered and deaerated with a $0.45-\mu \mathrm{m}$ nylon filter before use. Bath temperature was set at $37 \pm 0.5^{\circ} \mathrm{C}$. Ten-milliliter samples were drawn at each sampling time and replenished with $10 \mathrm{~mL}$ of fresh dissolution medium. Samples drawn were immediately centrifuged at $3500 \mathrm{rpm}$ and analyzed spectrophotometrically. Trials were performed with six tablets, and the mean values were used for data analysis.

Table 2 summarizes the conditions used for each drug product. Both strengths of ibuprofen tablets were assayed using the same experimental conditions.

A short validation program was performed for the four different spectrophotometric methods employed to determine the percent dissolved. Linearity, precision, and specificity were assessed for each combination of dissolution medium and wavelength.

\section{Data Analysis}

The dissolution profiles of all possible pairs of pharmaceutical equivalents were compared by similarity factor $f_{2}$ (22). The similarity factor is a logarithmic reciprocal square-root transformation of the sum of squared error and is a measurement of the similarity in the percent of dissolution between the two curves:

$$
f_{2}=50 \times \log \left\{\left[1+(1 / n) \sum_{t=t_{1}}^{t_{n}}\left(R_{t}-T_{t}\right)^{2}\right]^{-0.5} \times 100\right\}
$$


Table 2. Conditions Used for Each Drug Product According to USP (22)

\begin{tabular}{lcccc}
\hline & Dissolution Media - Volume & Apparatus -Speed & Sampling times (min) & Quantification wavelength (nm) \\
\hline Ranitidine & Water $-900 \mathrm{~mL}$ & 2 (paddle) $-50 \mathrm{rpm}$ & $5,15,30,45,60,90$ \\
\hline Furosemide & Phosphate buffer pH $5.8-900 \mathrm{~mL}$ & 2 (paddle) $-50 \mathrm{rpm}$ & $5,15,30,45,60,90$ \\
\hline lbuprofen & Phosphate buffer pH $7.2-900 \mathrm{~mL}$ & 2 (paddle) $-50 \mathrm{rpm}$ & $5,15,30,60,90$ \\
\hline Cephalexin & Water $-900 \mathrm{~mL}$ & 1 (basket, 40 -mesh cloth) - & 271 \\
\hline
\end{tabular}

All trials were performed with six tablets at $37 \pm 0.5^{\circ} \mathrm{C}$.

where $R_{t}$ and $T_{t}$ represent the percent dissolved at time $t$ for the reference and test products, respectively. We computed the $f_{2}$ value with the points of the dissolution profile up to the moment in which the reference product dissolved $85 \%$ or more. Therefore, the resulting matrixes are not symmetrical, since depending on the product taken as reference, the number of points of the dissolution profile considered for the comparison analysis might not be the same for the same pair of products.

We considered two products to be similar if they had similar dissolution profiles, and two dissolution profiles are considered similar when the $f_{2}$ value between them is greater than or equal to 50 . Although in vitro dissimilarity (i.e., dissimilarity between the dissolution profiles of two products) might not necessarily reflect an in vivo dissimilarity (absence of bioequivalence), at least in the case of some drugs (BCS Class II: highly permeable, poorly soluble), significant differences in the dissolution profiles will result in differences in bioavailability, since dissolution is the rate-determining step of the absorption process.

In the analysis of the results for each medication, we considered two types of possible deviations from the principle of transitivity: deviation type I, which corresponds to two products $B$ and $C$ that, having proved in vitro similarity to the reference product $A$ (the market leader or innovator), are not similar between themselves $\left(f_{2 \mathrm{BA}}\right.$ and $f_{2 \mathrm{CA}} \geq 50$, but $\left.f_{2 \mathrm{CB}}<50\right)$; and deviation type II, corresponding to the situation in which a given product $C$ is not equivalent to the reference product $A\left(f_{2 C A}<50\right)$ but is, however, similar to another product $B$ that is itself similar to $\mathrm{A}\left(f_{2 \mathrm{CB}} \geq 50\right)$.

We consider deviation type I to represent the most serious issue, since substitution of a product $B$ by a nonsimilar product $C$ may be recommended based on the fact that both $B$ and $C$ have proven bioequivalence to $A$. However, $B$ and $C$ are not similar in terms of dissolution

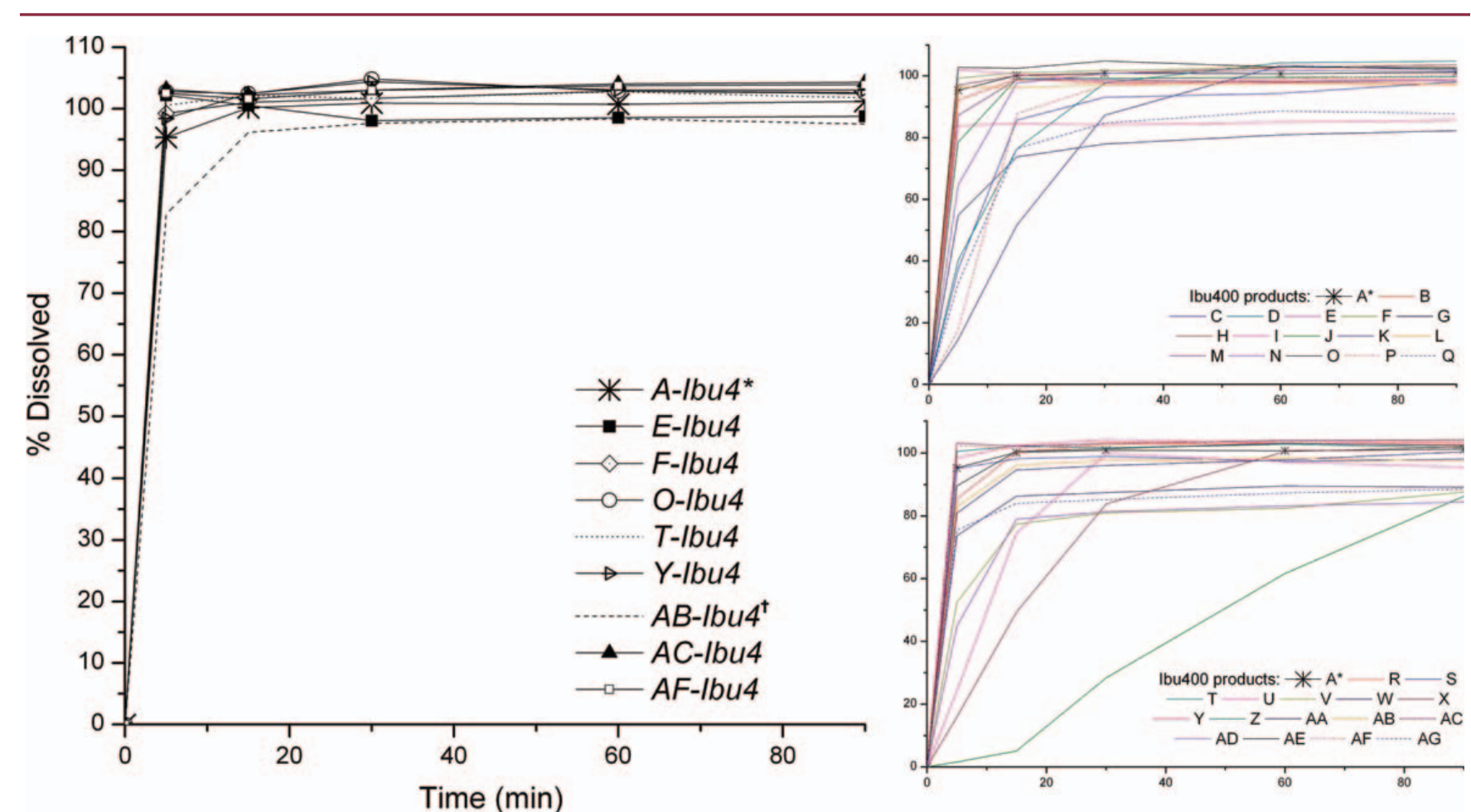

Figure 2. Dissolution profiles of ibuprofen 400-mg products (Apparatus 2, $50 \mathrm{rpm}, 900 \mathrm{~mL}$ of pH 7.2 phosphate buffer as dissolution medium). 


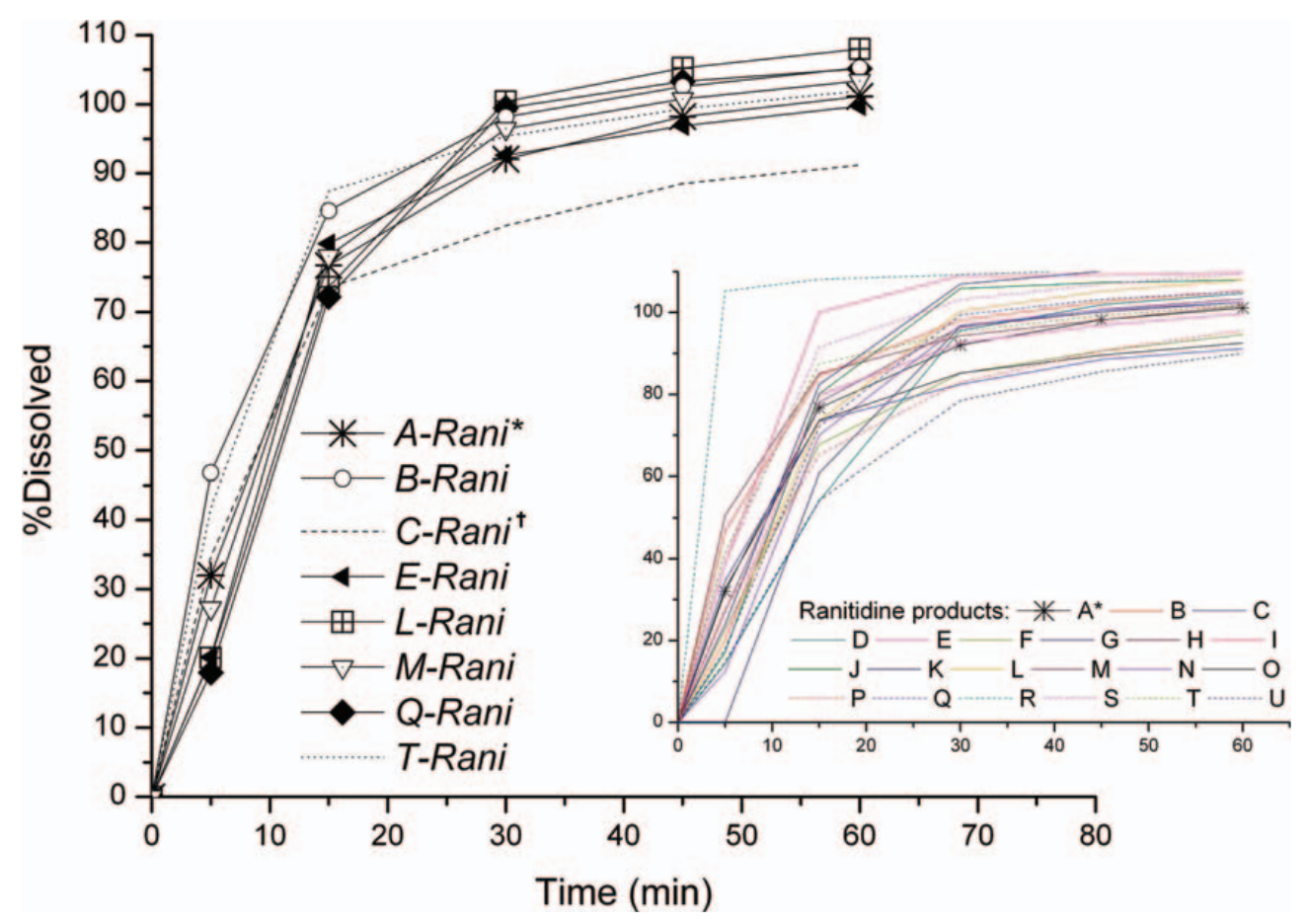

Figure 3. Dissolution profiles of ranitidine 300-mg products (Apparatus 2, $50 \mathrm{rpm}, 900 \mathrm{~mL}$ of water as dissolution medium).

profiles, and at least for some drugs, this may be reflected in differences in bioavailability. Deviation type II means only that two products that might be interchangeable are not considered for reciprocal substitution because one of them is not similar to the reference A. Therefore, deviation type I causes some extent of risk to the health of the patient (one product might not be as effective or safe as the other), while deviation type II only causes an economical issue.

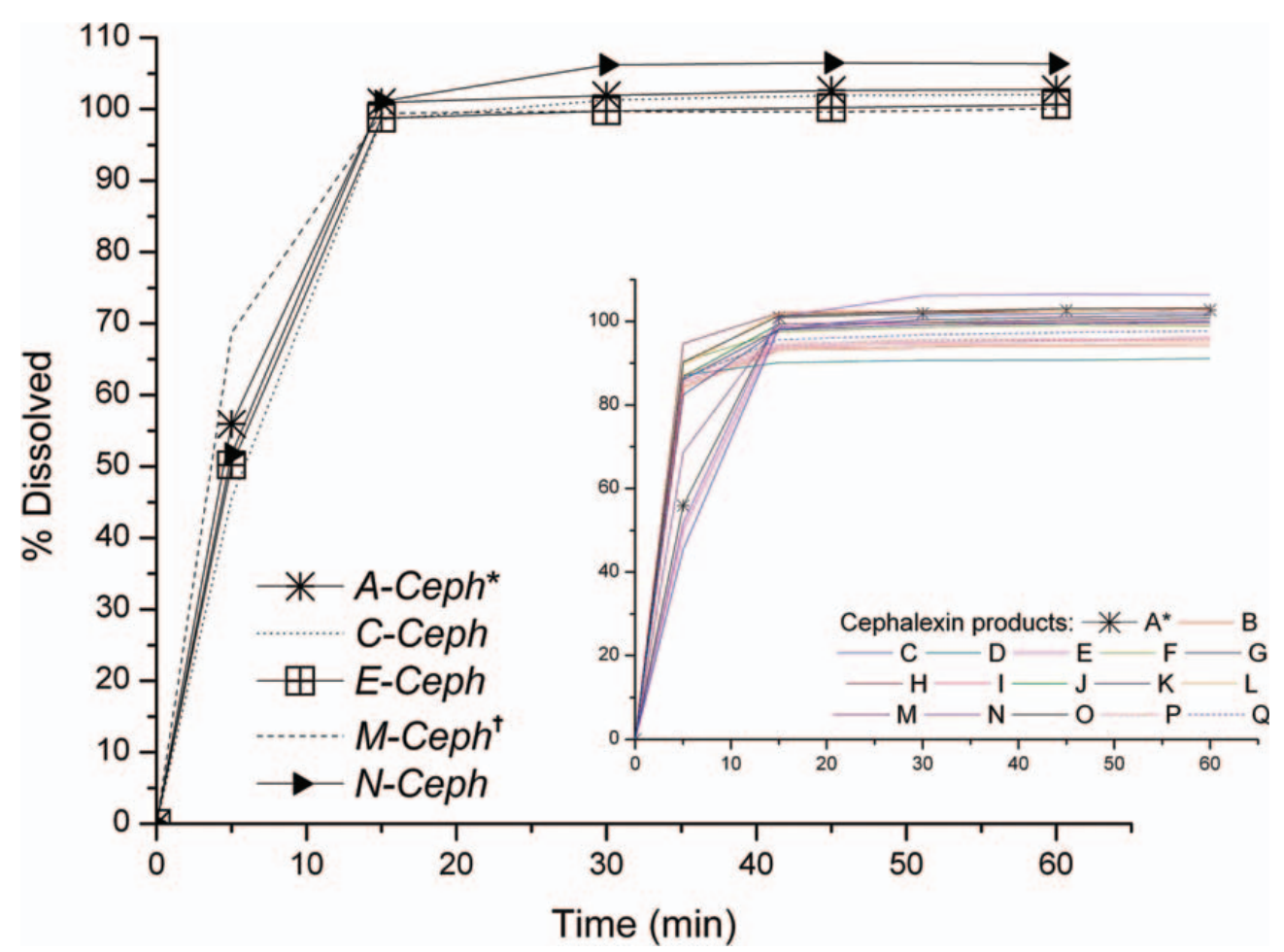

Figure 4. Dissolution profiles of cephalexin 500-mg products (Apparatus 1, $100 \mathrm{rpm}, 900 \mathrm{~mL}$ of water as dissolution medium). 


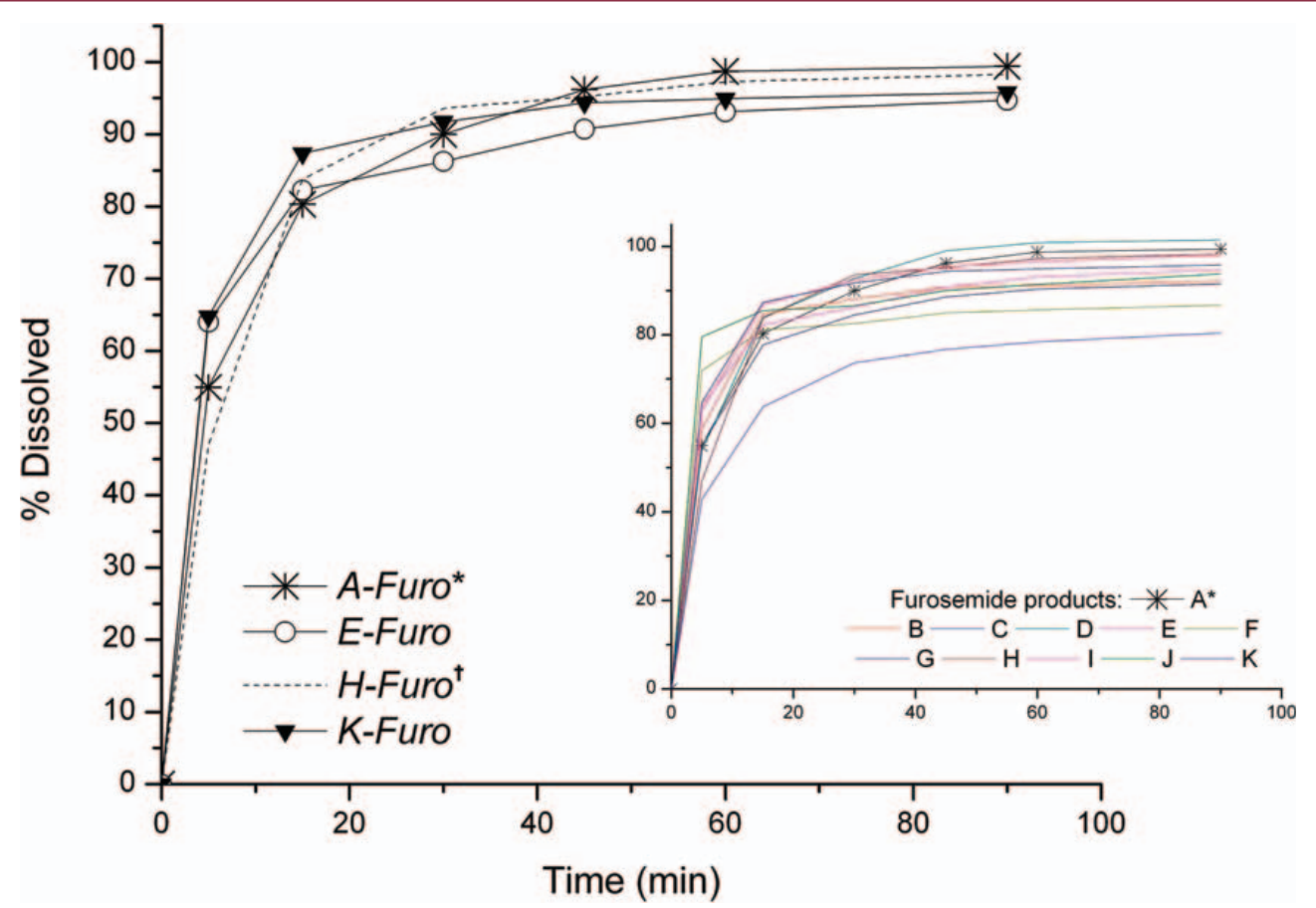

Figure 5. Dissolution profiles of furosemide 40-mg products (Apparatus 2, $50 \mathrm{rpm}, 900 \mathrm{~mL}$ of $\mathrm{pH} 5.8$ phosphate buffer as dissolution medium).

\section{RESULTS AND DISCUSSION}

Figures 2-6 present dissolution profiles for the products tested; examples of deviations from the transitivity principle are presented with grayscale lines, while the dissolution profiles of all the tested products for each drug are shown in small graphs with colored lines. The grayscale graphs of Figures $2-5$ present, for all the medications except ibuprofen $600 \mathrm{mg}$, the dissolution profiles of a certain product involved in type I deviations (denote with the ${ }^{\dagger}$ superscript), plus the profile of the corresponding reference product (denote with the * superscript) and all the products that present type I deviations from the

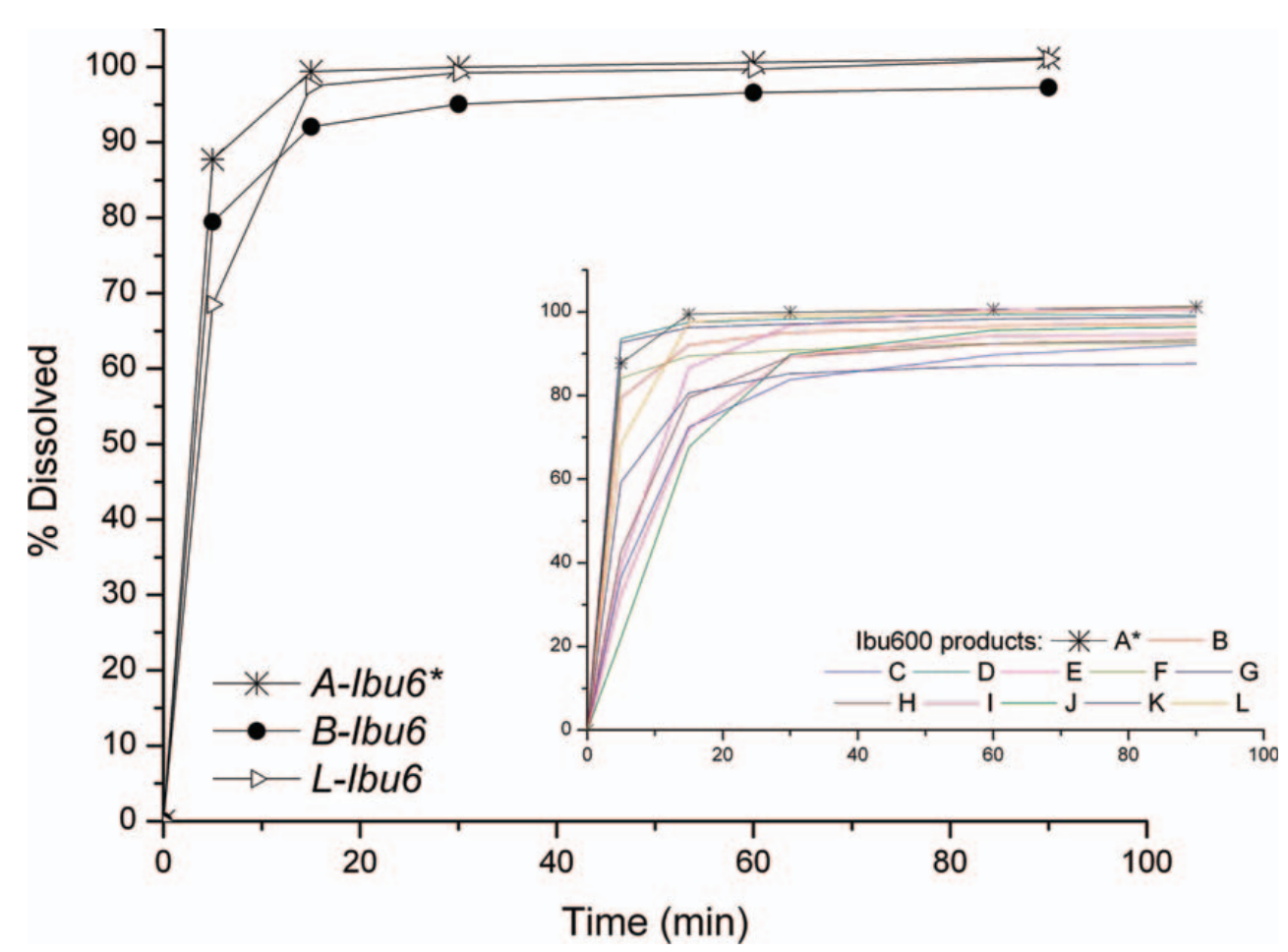

Figure 6. Dissolution profiles of ibuprofen 600-mg products (Apparatus 2, $50 \mathrm{rpm}, 900 \mathrm{~mL} \mathrm{of} \mathrm{pH} 7.2$ phosphate buffer as dissolution medium). 


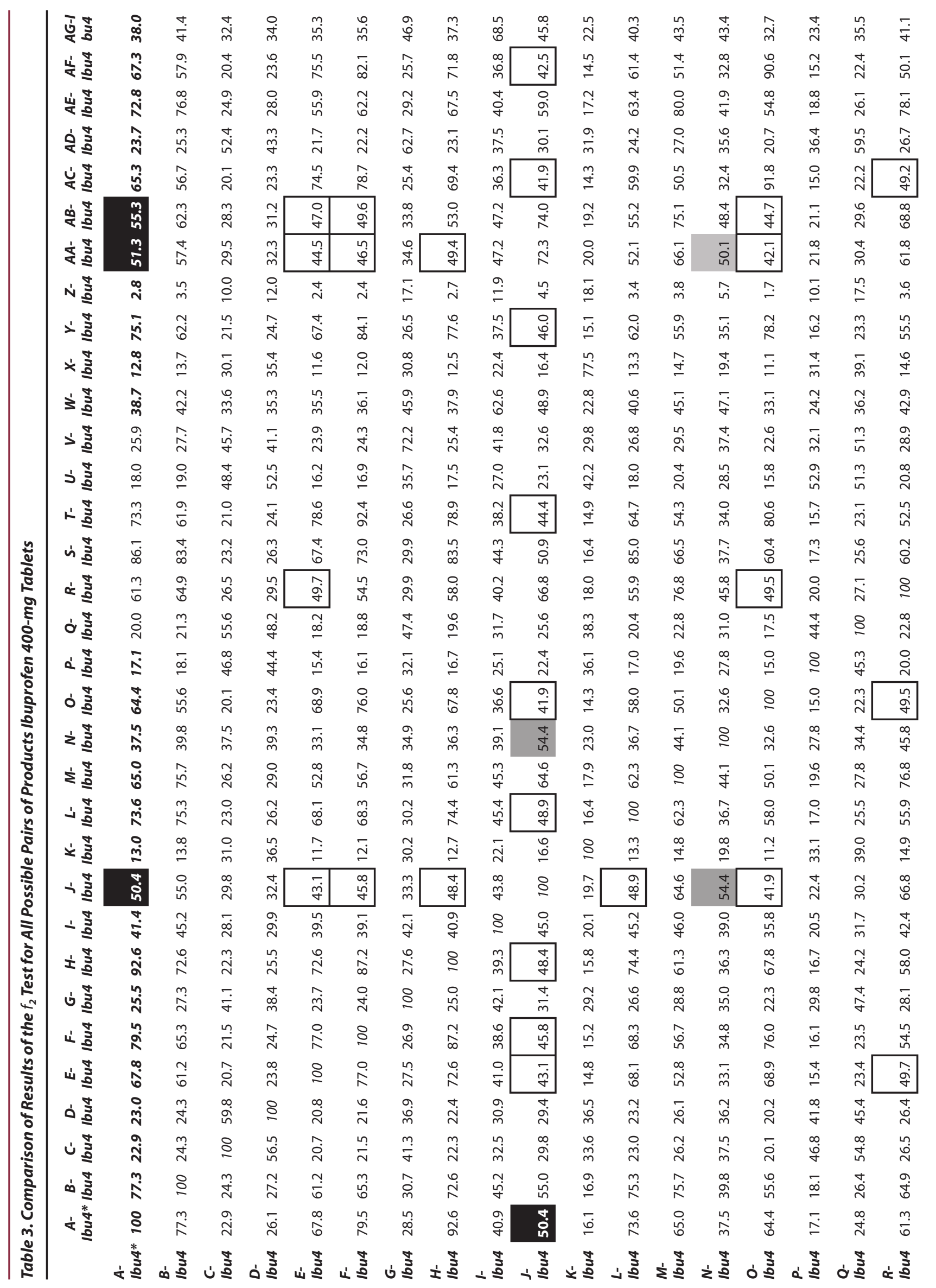

Dissolution Technologies | FEBRUARY 2012 


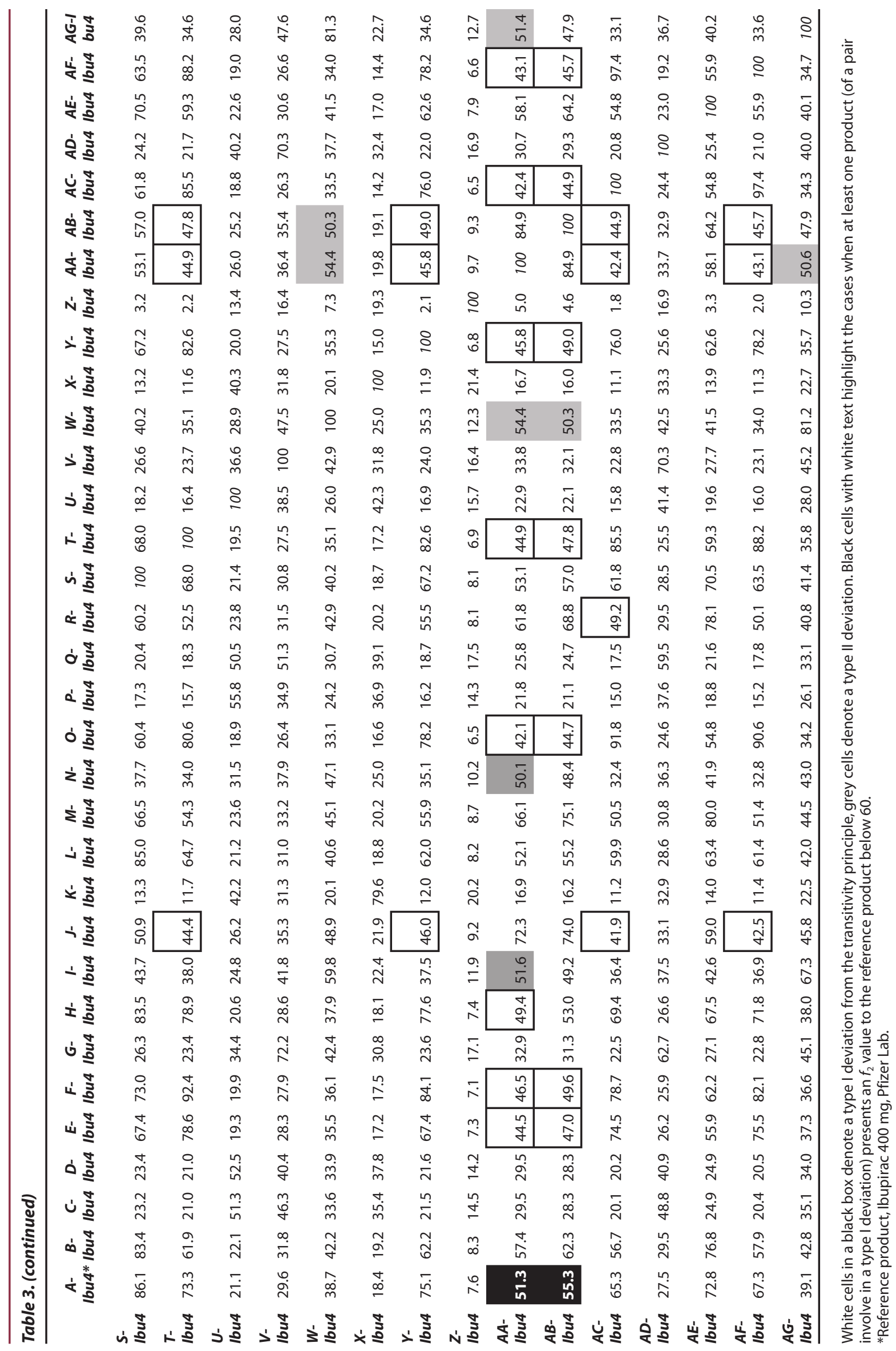


Table 4. Comparison of Results of the $f_{2}$ Test for All Possible Pairs of Products Ibuprofen 600-mg Tablets

\begin{tabular}{|c|c|c|c|c|c|c|c|c|c|c|c|c|}
\hline & A-Ibu6* & B-Ibu6 & C-Ibu6 & D-Ibu6 & E-Ibu6 & F-Ibu6 & G-Ibu6 & H-Ibu6 & I-Ibu6 & J-Ibu6 & K-Ibu6 & L-Ibu6 \\
\hline A-Ibu6* & 100 & 57.6 & 23.0 & 71.0 & 26.9 & 54.4 & 70.3 & 26.8 & 22.0 & 18.5 & 33.5 & 47.5 \\
\hline B-Ibu6 & 57.6 & 100 & 27.6 & 52.3 & 31.6 & 69.3 & 54.4 & 32.2 & 26.3 & 22.1 & 41.9 & 56.2 \\
\hline C-Ibu6 & 27.9 & 32.8 & 100 & 26.8 & 48.6 & 32.0 & 27.4 & 64.5 & 69.9 & 54.4 & 47.7 & 34.6 \\
\hline D-Ibu6 & 71.0 & 52.3 & 21.6 & 100 & 24.8 & 53.7 & 91.0 & 25.0 & 20.6 & 17.3 & 31.6 & 41.9 \\
\hline E-Ibu6 & 26.9 & 31.6 & 47.5 & 24.8 & 100 & 29.1 & 25.2 & 59.9 & 49.2 & 40.6 & 43.1 & 37.2 \\
\hline F-Ibu6 & 54.4 & 69.3 & 26.5 & 53.7 & 29.1 & 100 & 56.7 & 30.4 & 25.0 & 21.0 & 40.4 & 47.3 \\
\hline G-Ibu6 & 70.3 & 54.4 & 22.2 & 91.0 & 25.2 & 56.7 & 100 & 25.6 & 21.1 & 17.7 & 32.5 & 42.6 \\
\hline H-Ibu6 & 29.7 & 35.2 & 62.3 & 28.0 & 58.0 & 33.5 & 28.6 & 100 & 59.1 & 45.8 & 52.3 & 38.6 \\
\hline I-Ibu6 & 25.0 & 29.4 & 68.5 & 23.7 & 51.0 & 28.1 & 24.2 & 59.1 & 100 & 62.0 & 41.5 & 32.0 \\
\hline J-Ibu6 & 21.6 & 25.2 & 52.7 & 20.4 & 43.3 & 24.1 & 20.8 & 45.8 & 62.0 & 100 & 34.5 & 27.5 \\
\hline K-Ibu6 & 35.2 & 43.5 & 45.6 & 33.8 & 43.1 & 43.1 & 34.8 & 52.3 & 41.5 & 34.5 & 100 & 43.6 \\
\hline L-Ibu6 & 47.5 & 56.2 & 30.0 & 41.9 & 37.2 & 47.3 & 42.6 & 36.0 & 29.1 & 24.5 & 43.1 & 100 \\
\hline
\end{tabular}

Grey cells denote a type II deviation.

* Reference product, Ibupirac 600 mg, Pfizer Lab.

transitivity principle with the considered product $\left({ }^{\dagger}\right)$. For example, Figure 2 shows the dissolution profile of ibuprofen $400 \mathrm{mg}$ product $A B\left(A B-l b u 4^{\dagger}\right)$, plus those of the reference product $\left(A-I b u 4^{*}\right)$ and all the products involved in type I deviations with $A B-1 b u 4^{\dagger}$ (E-Ibu4, F-lbu4, O-Ibu4, $T$-Ibu4, Y-Ibu4, AC-Ibu4 and AF-Ibu4). For ibuprofen $600 \mathrm{mg}$ (Figure 6), because no type I deviations are observed, the only type Il deviation found is shown. Note that in all the cases presented here, the deviations from the transitivity principle involve, circumstantially, products that dissolve well in the media used (more than $85 \%$ dissolved in the first $30 \mathrm{~min}$ ). This is a consequence of the fact that, in all cases, the reference product dissolves well. However, if any reference product had poor dissolution behavior, the dissolution profile would show deviations from the transitivity principle. This is natural since, from our definitions of deviations type I and type II from the transitivity principle, at least one of the two generic products involved in a deviation has to be similar to the reference product.

Tables 3-7 present the $f_{2}$ values for all the possible pairs of products that can be considered. The product in the first column is taken as reference for each comparison (see $f_{2}$ eq). The first row of each table corresponds to the reference product established by the Argentinean National Health Authority (ANMAT). When no reference has been defined by such authority, we considered the market leader as the reference product. White cells in a black box denote a type I deviation from the transitivity principle, as defined in the Data Analysis section. Grey cells denote a type II deviation from the transitivity principle. We have only considered the actual reference product (the one defined by the ANMAT or the market leader). However, many more deviations from the transitivity principle may arise if other reference products were considered.

It is noteworthy that several deviations from the transitivity principle (in total, 59 type I and 35 type II) have been observed. Let us consider, for the analysis of the results, an $n_{i} \times n_{i}$ matrix representing the entries of the table corresponding to the medication $i, n_{i}$ being the number of brands in the Argentinean market for that medication. We can calculate the percentage of exceptions to the principle for each table as

$$
\text { Total\% exceptions }=\frac{\sum_{i} \text { Number of exceptions }}{\sum_{i}\left[\frac{n_{i}^{2}-n_{i}}{2}-\left(n_{i}-1\right)\right]} \times 100
$$

Note that $\left(n_{i}^{2}-n_{i}\right) / 2$ is the number of off-diagonal elements below the diagonal (in general, if the table element $x_{k j}$ is marked as an exception so will be $x_{j k}$; what is more, we are not interested in those cases when $j=k$ ). We have also extracted from the denominator those $\left(n_{i}-1\right)$ elements below the diagonal that correspond to a comparison between any given product and the reference, since discussion of the principle of transitivity when the reference is one of the compounds being compared is irrelevant. The calculated percent of type I deviations is $6.5 \%$ and that of type II deviations is 3.9\% (over the total comparisons). It is again worth highlighting that we have only considered exceptions to the principle of transitivity when actual reference products for bioequivalence studies indicated by the Argentinean health authority are used as references. If other products were considered as references, many more exceptions would be found. 


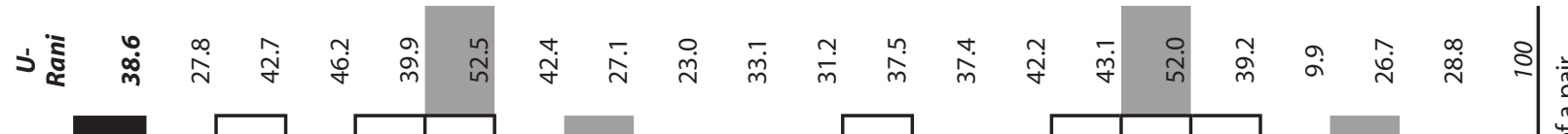

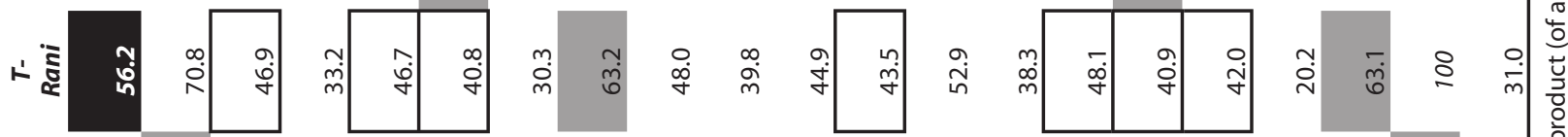

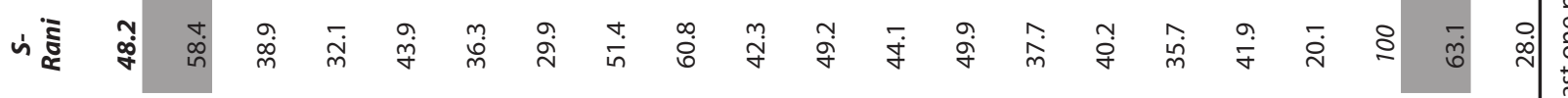

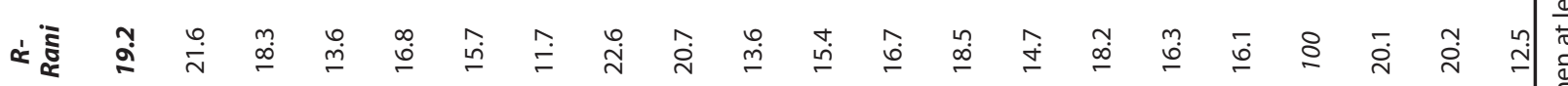

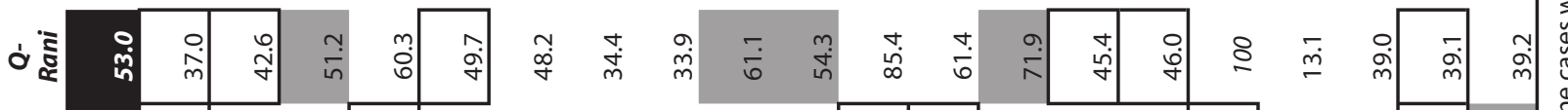

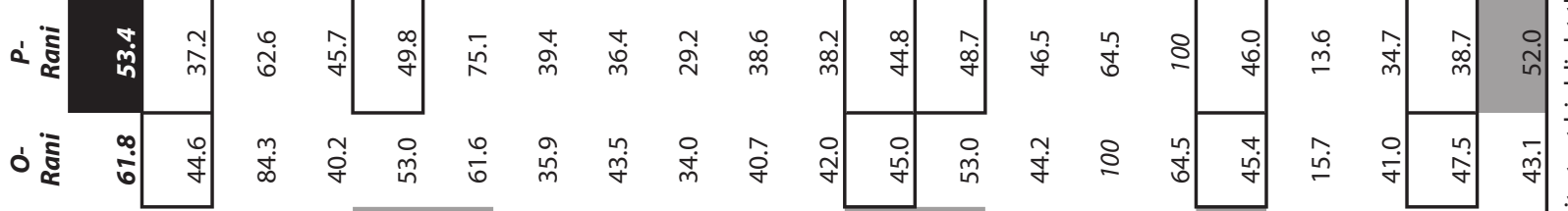

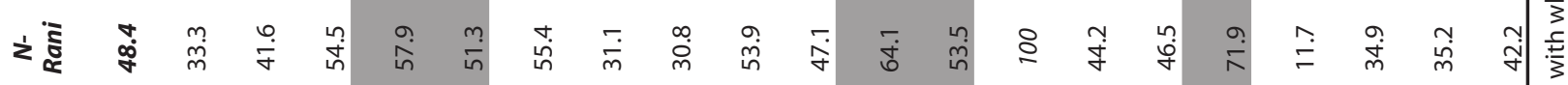

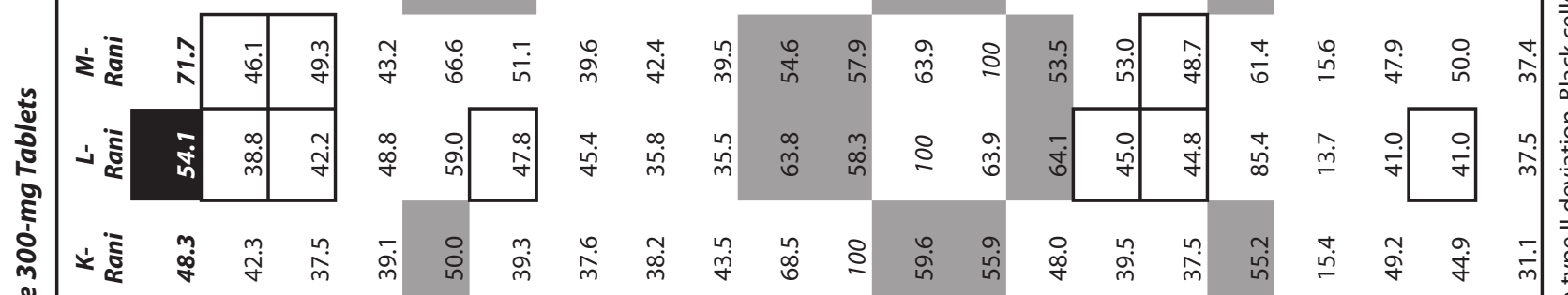

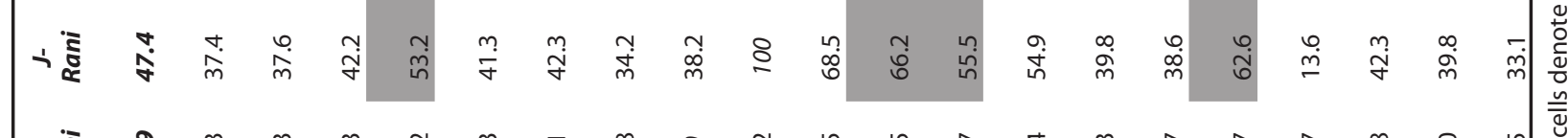

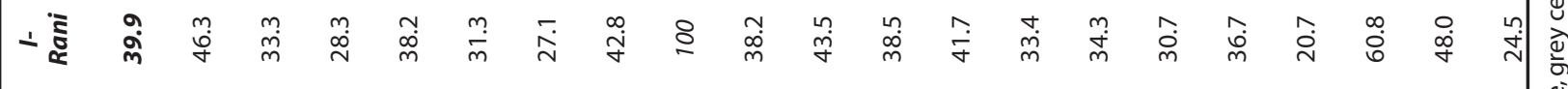

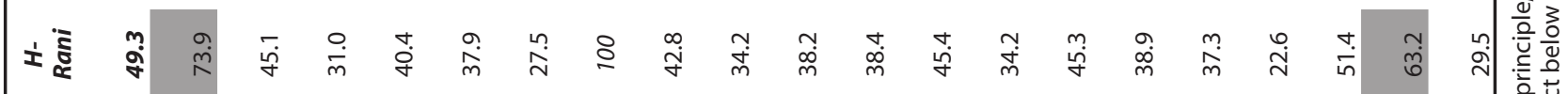

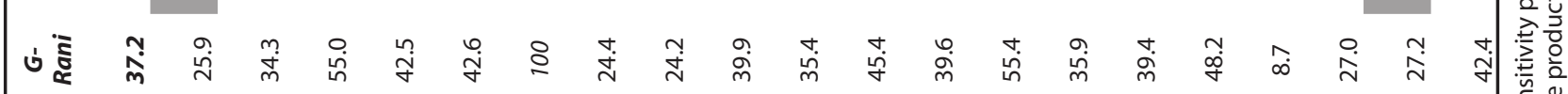

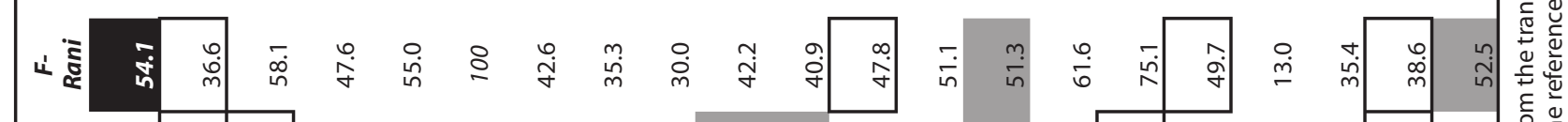

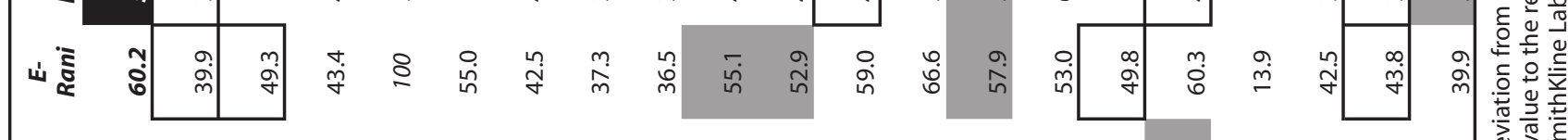

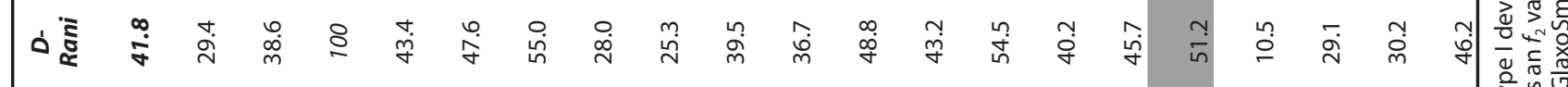

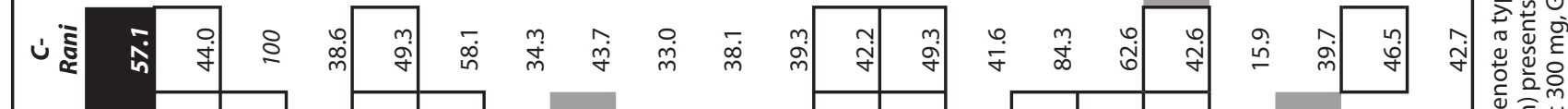

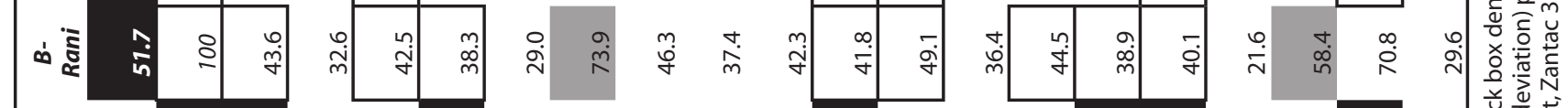

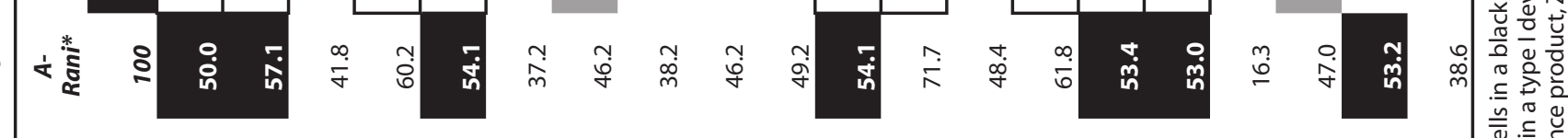

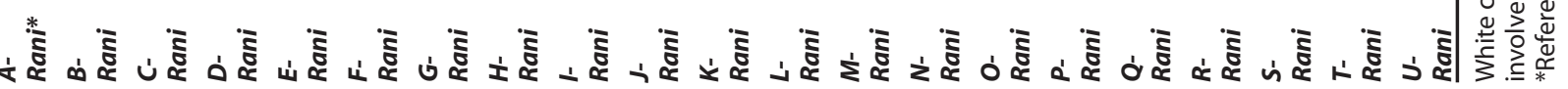




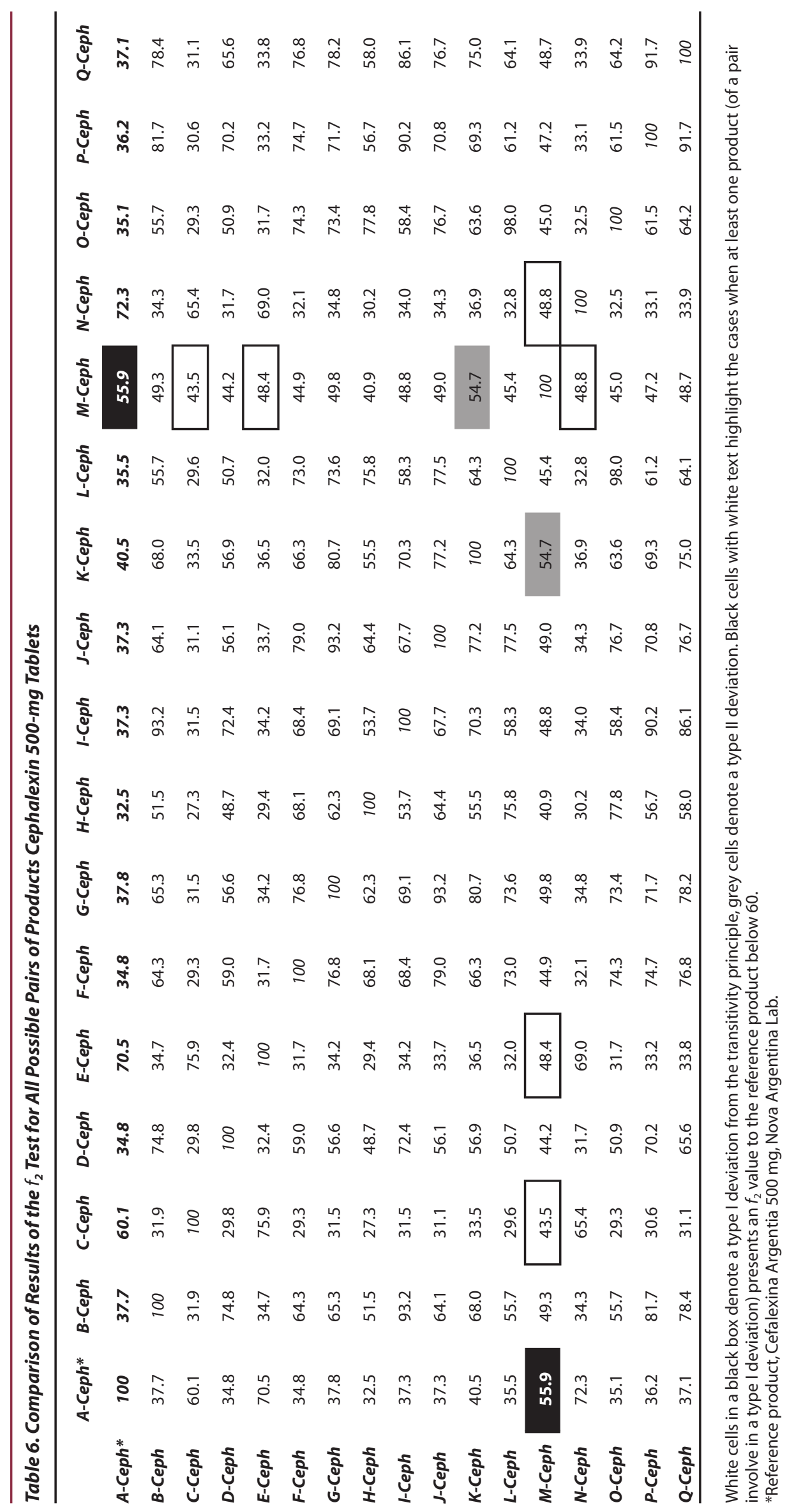


Table 7. Comparison of Results of the $f_{2}$ Test for All Possible Pairs of Products Furosemide 40-mg Tablets

\begin{tabular}{|c|c|c|c|c|c|c|c|c|c|c|c|}
\hline & A-Furo* & B-Furo & C-Furo & D-Furo & E-Furo & F-Furo & G-Furo & H-Furo & I-Furo & J-Furo & K-Furo \\
\hline A-Furo* & 100 & 71.9 & 41.0 & 77.0 & 61.6 & 48.4 & 72.1 & 62.9 & 60.4 & 41.6 & 57.4 \\
\hline B-Furo & 71.9 & 100 & 38.0 & 69.8 & 71.6 & 53.3 & 64.7 & 56.1 & 71.0 & 45.8 & 67.6 \\
\hline C-Furo & 38.2 & 39.9 & 100 & 35.5 & 39.1 & 39.2 & 45.6 & 37.9 & 35.1 & 33.2 & 35.2 \\
\hline D-Furo & 77.0 & 69.8 & 38.1 & 100 & 57.5 & 45.8 & 60.8 & 69.3 & 62.7 & 40.7 & 58.7 \\
\hline E-Furo & 61.6 & 71.6 & 37.5 & 57.5 & 100 & 64.3 & 61.4 & 48.4 & 66.7 & 51.7 & 67.5 \\
\hline F-Furo & 48.4 & 53.3 & 34.6 & 45.8 & 64.3 & 100 & 50.0 & 40.0 & 53.6 & 62.5 & 55.8 \\
\hline G-Furo & 65.3 & 67.0 & 45.4 & 56.5 & 64.0 & 52.5 & 100 & 55.9 & 54.8 & 44.4 & 54.1 \\
\hline H-Furo & 62.9 & 56.1 & 39.1 & 69.3 & 48.4 & 40.0 & 55.1 & 100 & 51.3 & 35.7 & 48.8 \\
\hline I-Furo & 60.4 & 71.0 & 34.1 & 62.7 & 66.7 & 53.6 & 53.9 & 51.3 & 100 & 49.4 & 90.2 \\
\hline J-Furo & 41.6 & 45.8 & 29.4 & 40.7 & 51.7 & 62.5 & 41.4 & 35.7 & 49.4 & 100 & 51.9 \\
\hline K-Furo & 57.4 & 67.6 & 33.5 & 58.7 & 67.5 & 55.8 & 52.4 & 48.8 & 90.2 & 51.9 & 100 \\
\hline
\end{tabular}

White cells in a black box denote a type I deviation from the transitivity principle, grey cells denote a type Il deviation. Black cells with white text highlight the cases when at least one product (of a pair involve in a type I deviation) presents an $f_{2}$ value to the reference product below 60 .

*Reference product, Lasix 40 mg, Sanofi-Aventis Lab.

If many exceptions to the transitivity principle are observed in vitro through dissolution profiles, it is probable that deviations from the principle would be observed in bioequivalence studies, at least for BCS Class II drugs in which dissolution is the rate-limiting step of the absorption process (e.g., ibuprofen, naproxen) (23). It is also interesting that $93 \%$ of the type I deviations include at least one product with $f_{2}$ to the reference product (black cells with white text in the tables) less than 60 (i.e., near the threshold value of 50). Also note that many deviations from the transitivity principle involve drugs that have been proposed as candidates for biowaivers or have already been granted biowaivers (e.g., ibuprofen and ranitidine).

\section{CONCLUSIONS}

Although the transitivity principle is assumed to rule in bioequivalence studies, our findings support previous findings by Benet and Goyan (18), Anderson and Hauck (19), and Midha et al. (20) regarding the non-general applicability of transitivity to define medication substitutions by pharmaceutical equivalents. Therefore, the only safe and effective substitution that may be suggested from a bioequivalence study is the substitution of reference product $A$ by products for which bioequivalence has been demonstrated (e.g., products $B$ and $C$ ). Bioequivalence of $B$ and $C$ is not granted from $a$ bioequivalence study to reference $A$.

Potential deviations from the transitivity principle should be specially considered for (1) highly permeable drugs for which dissolution is the rate-limiting step of the absorption process (Class II of the BCS) and (2) drugs with a narrow therapeutic window, for which small differences in bioavailability may produce important therapeutic differences.

Because most of the observed deviations from the transitivity principle involve pairs of products with $f_{2}$ values near the threshold of 50 , a redefinition of this threshold value (e.g., 60) may lead to a lower frequency of deviations.

Alternatively, public health authorities could require private pharmaceutical companies to prove bioequivalence not only to a reference product (innovator or lead product) but to all products that exist in the market and to make this information available to the public to provide physicians and pharmacists with safer criteria for rational substitution/interchangeability. Alternatively, governments could finance these bioequivalence studies. This would mean a great investment from the private pharmaceutical sector or from the public health offices but an important (and safe) saving on medications for patients, providing a wider offering of potential substitutions, since many pairs of products with similar dissolution profiles-and thus, candidates for interchangeability - are not similar to the innovator product.

\section{ACKNOWLEDGMENTS}

A. Talevi is an Assistant Researcher from CONICET. A. Gregorini is a fellow of CIC. The authors are grateful to the Pharmaceutical College of La Plata for the funding of this work.

\section{REFERENCES}

1. The World Medicines Situation;WHO/EDM/PAR/2004.5; World Health Organization: Géneva, 2004. www.ops. org.bo/textocompleto/ime23901.pdf (accessed Jan 12, 2012). 
2. Herrera, M. M. C.; Picos, D. R.; Vega, R.Y.; Pérez, B. de la C. Should the Control of Drug Prices Be a Responsibility of the National Regulatory Authorities? Lat. Am. J. Pharm. 2007, 26 (6), 913-923.

3. Savings From Generic Drugs Purchased at Retail Pharmacies, 2004. U.S. Food and Drug Administration Web site. http://www.fda.gov/Drugs/ EmergencyPreparedness/BioterrorismandDrugPrepar edness/ucm134205.htm (accessed Jan 19, 2012).

4. Generic Drugs: Questions and Answers. U.S. Food and Drug Administration Web site. www.fda.gov/drugs/ resourcesforyou/consumers/questionsanswers/ ucm 100100.htm (accessed Jan 19, 2012).

5. de Joncheere, K.; Rietveld, A. H.; Huttin, C. Experiences with generics. In Drugs and Money. Prices, affordability and cost containment, 7th ed.; Dukes, M. N. G., Haaijer-Ruskamp, F. M., de Joncheere, C. P., Rietveld, A. H., Eds.; World Health Organization: Geneva, 2003; pp 101-110.

6. How Increased Competition from Generic Drugs Has Affected Prices and Returns in the Pharmaceutical Industry; A CBO Study; U.S. Congressional Budget Office, The Congress of the United States, U.S. Government Printing Office:Washington, DC, 1998.

7. Bioavailability and Bioequivalence Studies for Orally Administered Drug Products—General Considerations; Guidance for Industry; U.S. Department of Health and Human Services, Food and Drug Administration, Center for Drug Evaluation and Research (CDER), U.S. Government Printing Office:Washington, DC, 2003.

8. Note for Guidance on the Investigation of Bioavailability and Bioequivalence; CPMP/EWP/QWP/1401/98; Committee for Proprietary Medicinal Products (CPMP), European Medicines Agency: London, 2001.

9. Waiver of In Vivo Bioavailability and Bioequivalence Studies for Immediate-Release Solid Oral Dosage Forms Based on a Biopharmaceutics Classification System; Guidance for Industry; U.S. Department of Health and Human Services, Food and Drug Administration, Center for Drug Evaluation and Research (CDER), U.S. Government Printing Office:Washington, DC, 2000.

10. Tsume, Y.; Amidon, G. L. The biowaiver extension for BCS class III drugs: the effect of dissolution rate on the bioequivalence of BCS class III immediate-release drugs predicted by computer simulation. Mol. Pharm. 2010, 7 (4), 1235-1243.

11. Tubic-Grozdanis, M.; Bolger, M. B.; Langguth, P. Application of Gastrointestinal Simulation for Extensions for Biowaivers of Highly Permeable Compounds. AAPS J. 2008, 10 (1), 213-226.
12. Graffner, C. Regulatory aspects of drug dissolution from a European perspective. Eur. J. Pharm. Sci. 2006, 29 (3-4), 288-293.

13. Yazdanian, M.; Briggs, K.; Jankovsky, C.; Hawi, A.The "High Solubility" Definition of the Current FDA Guidance on Biopharmaceutical Classification System May Be Too Strict for Acidic Drugs. Pharm. Res. 2004, 21 (2), 293-299.

14. Potthast, H.; Dressman, J. B.; Junginger, H. E.; Midha, K. K.; Oeser, H.; Shah, V. P.; Vogelpoel, H.; Barends, D. M. Biowaiver monographs for immediate release solid oral dosage forms: ibuprofen. J. Pharm. Sci. 2005, 94 (10), 2121-2131.

15. Kortejarvi, H.; Yliperttula, M.; Dressman, J. B.; Junginger, H. E.; Midha, K. K.; Shah, V. P.; Barends, D. M. Biowaiver monographs for immediate release solid oral dosage forms: ranitidine hydrochloride. J. Pharm. Sci. 2005, 94 (8), 1617-1625.

16. Pharmaceutical Development for Multisource (Generic) Pharmaceutical Products; Working document QAS/08.251/Rev.1; World Health Organization: Geneva, 2010.www.who.int/medicines/services/ expertcommittees/pharmprep/ 150510-PharmDevelGener_QAS08_251Rev1.pdf (accessed Jan 15, 2012).

17. World Health Organization Expert Committee on Specifications for Pharmaceutical Preparations; WHO Technical Report Series, No. 937; World Health Organization: Geneva, 2006.

18. Benet, L. Z.; Goyan, J. E. Bioequivalence and narrow therapeutic index drugs. Pharmacotherapy 1995, 15 (4), 433-440.

19. Anderson, S.; Hauck, W.W.The transitivity of bioequivalence testing: potential for drift. Int. J. Clin. Pharmacol. Ther. 1996, 34 (9), 369-374.

20. Midha, K. K.; Rawson, M. J.; Hubbard, J.W. Bioequivalence: Switchability and scaling. Eur. J. Pharm. Sci. 1998, 6 (2), 87-91.

21. The United States Pharmacopeia and National Formulary USP 31-NF 26; The United States Pharmacopeial Convention, Inc.: Rockville, MD, 2008.

22. Dissolution Testing of Immediate Release Solid Oral Dosage Forms; Guidance for Industry; U.S. Department of Health and Human Services, Food and Drug Administration, Center for Drug Evaluation and Research (CDER), U.S. Government Printing Office: Washington, DC, 1997.

23. Ruiz, M. E.; Rubini, A.; Mandel, J. S;Volonté, M. G. Pharmaceutical equivalence of naproxen $500 \mathrm{mg}$ tablets. Lat. Am. J. Pharm. 2007, 26 (4), 530-535. 